Research Paper

\title{
Clinical application of a novel endoscopic mask: A randomized controlled trial in aged patients undergoing painless gastroscopy
}

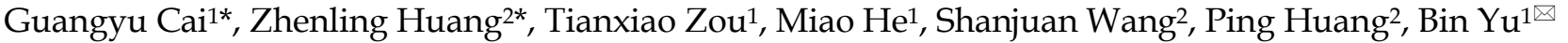 \\ 1. Department of Anesthesiology, Shanghai Tongji Hospital, Tongji University School of Medicine, Shanghai, China; \\ 2. Department of Anesthesiology, Renji Hospital affiliated to School of Medicine, Shanghai Jiao Tong University, Shanghai, China. \\ *Equal contributors. \\ $\triangle$ Corresponding author: Bin Yu, Department of Anesthesiology, Shanghai Tongji Hospital, Tongii University School of Medicine, NO.389, Xincun Road, Putuo \\ District, Shanghai, China, 13918108880, E-mail: yubin@tongji.edu.cn. \\ (c) Ivyspring International Publisher. This is an open access article distributed under the terms of the Creative Commons Attribution (CC BY-NC) license \\ (https://creativecommons.org/licenses/by-nc/4.0/). See http://ivyspring.com/terms for full terms and conditions.
}

Received: 2016.07.21; Accepted: 2016.12.20; Published: 2017.02.08

\begin{abstract}
Background: Desaturation during painless gastroscopy in aged patients leads to discontinuation of the procedure, prolonged manipulation time and increased risk of severe complications. An endoscopic nasal mask was designed to control hypoxia during the above procedures. A randomized trial was performed to test whether the novel endoscopic mask is helpful for hypoxia during painless gastroscopy in aged patients.

Methods: In this randomized, controlled trial, 141 aged patients undergoing painless gastroscopy were randomized into nasal catheter group (69 patients) and endoscopic mask group (65 patients). Primary outcomes were minimum pulse oxygen saturation and incidence of pulse oxygen saturation $\leq 90 \%$.

Results: Finally, 134 aged patients were analyzed, including 69 patients in nasal catheter group and 65 patients endoscopic mask group. The minimum pulse oxygen saturation $(96.4 \% \pm 4.8 \%)$ was higher in the aged endoscopic mask group than in the aged nasal catheter group $(94.3 \% \pm 5.6 \%, P$ $=0.0075)$. The incidence of pulse oxygen saturation $\leq 90 \%$ did not significantly differ between the endoscopic mask group and nasal catheter group $(6.2 \%$ VS $15.9 \%, P=0.07)$. There were no severe adverse events in either groups.

Conclusion: The endoscopic mask was safely used in aged patients during painless gastroscopy under propofol sedation and significantly improved the minimum pulse oxygen saturation without increasing time to examination or recovery time.
\end{abstract}

Key words: gastroscopy, mask, aged, propofol.

\section{Introduction}

Propofol, a short-acting intravenous anaesthetic, is widely used in painless gastroscopy because it is associated with rapid effects and rapid recovery from anesthesia[1]. However, owing to individual differences in the tolerance to anaesthetics, some patients may display a lower depth of anesthesia or delayed recovery from anesthesia after gastrointestinal endoscopic examination. Furthermore, propofol occasionally causes respiratory depression, resulting in hypoxia, decreasing of SPO2 and discontinuation of the procedure $[1,2]$. Tongue retraction after propofol-induced anesthesia can result in respiratory depression and respiratory tract obstruction, and decrease pulse oxygen saturation[3, 4]. In particular, aged patients with cardiovascular diseases or decreased cardiopulmonary functional reserve are prone to developing severe complications because of anesthesia-induced hypoxia[5, 6]. 
In this study, a novel multifunctional endoscopic mask was designed to provide oxygen and prevent hypoxia during painless gastroscopy (Fig. 1 and Fig. 2). We estimated the effects of this mask on minimum pulse oxygen saturation, incidence of adverse reactions, number of intubation attempts, time to intubation or examination, recovery time and propofol dosage in patients undergoing painless gastroscopy.

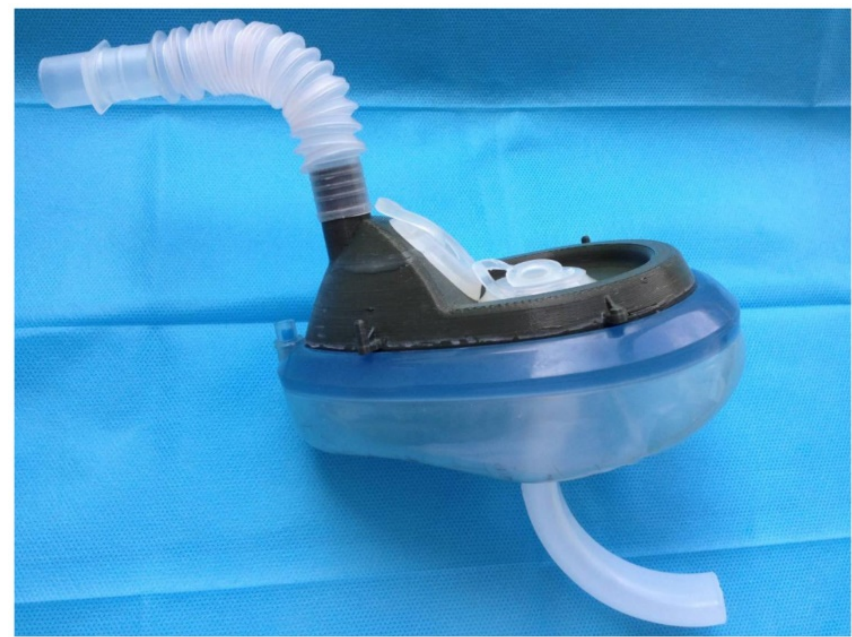

Figure 1. The endoscopic mask used in this study.

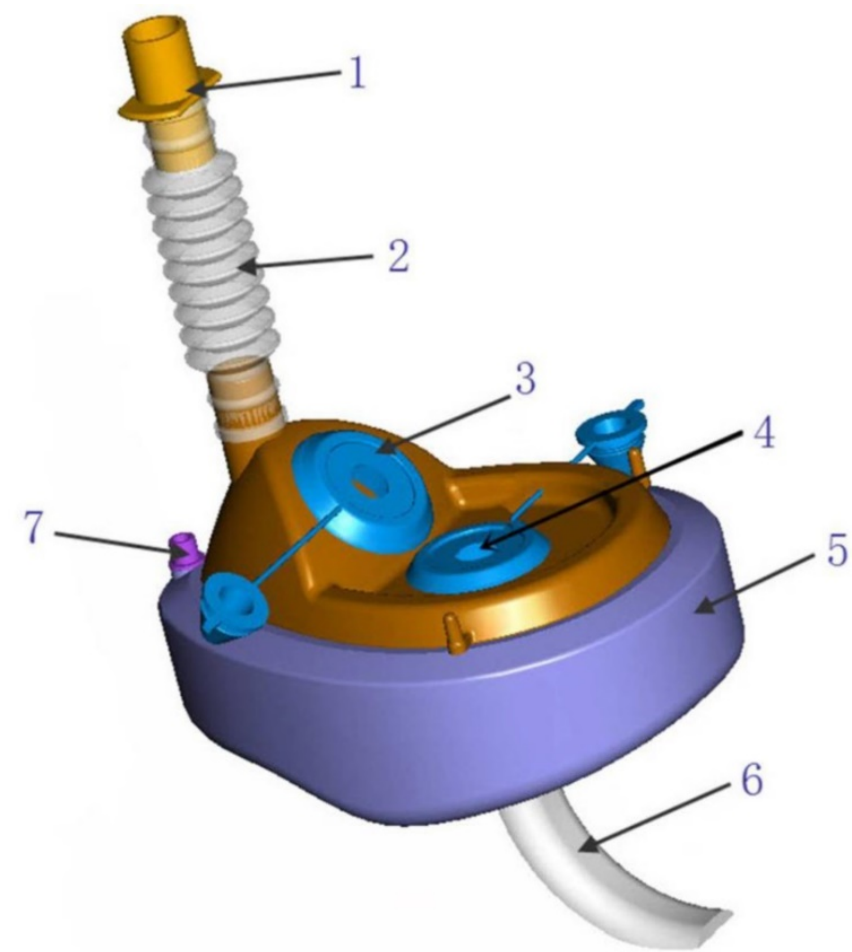

Figure 2. Pictorial depiction of the mask: (1) respiratory interface, (2) bellows, (3) nasal opening, (4) oral opening, (5) gasbag, (6) oropharyngeal airway and (7) one-way valve.

\section{Material and methods}

This randomized, controlled, single-blind trial was approved by the ethics committee of Shanghai Tongji Hospital (no. 199), and registered in the Chinese Clinical Trial Registry (http://www. chictr.org.cn; registration no.: ChiCTR-TRC-13004086; principal investigator: Bin $\mathrm{Yu}$; date of registration:8th, Sep, 2013). The subjects were patients who underwent painless gastroscopy on an outpatient basis in Shanghai Tongji Hospital and Shanghai Renji Hospital. All subjects provided written informed consent before participating in the study. Eligible subjects aged 65-80 years with a body mass index of $\leq 25$ had American Society Anesthesiologists physical status scores of I or II, with no serious cardiopulmonary or kidney diseases. Exclusion criteria are: 1) Serious coronary heart disease and myocardial injury with serious heart failure; 2) Stenosis of the esophagus or cardia obstruction; 3) Aortic aneurysm; 4) Hemorrhagic shock; 5) Acute pharyngitis and tonsillitis; 6) Pneumonia or other infectious fever; 7) Asthmatic breathing difficulties; 8) Severe pulmonary dysfunction; 9) Physical extreme weakness; 10) Patients did not cooperate or spirit was not normal; 11) Acute upper gastrointestinal bleeding; 12) Allergic to propofol or the emulsifier content; 13) Pregnancy and lactation women; 14) Severe sleep apnea syndrome. All experiments were conducted in gastroscope room with a standard anesthesia workstation. An experienced anesthetist cared for the subjects and managed any emergency situations with professional resuscitation equipment.

The gastroscope was passed into the esophagus or stomach via the oral opening of the endoscopic mask (PRC patent, ZL2012 1 0286504.5). During the procedure, the oxygen-flow rate was set at $5 \mathrm{~L} / \mathrm{min}$. The respiratory interface, which was connected to the oxygen supply equipment, was used for first-aid measures if hypoxia occurred.

The study was a randomized, controlled, single blind trial(patient-blinded), and subjects aged 65-80 years were randomized into nasal catheter group and endoscopic mask group. Eligible subjects scheduled to undergo painless gastroscopy were fasted for $12 \mathrm{~h}$ before the start of the examination, and their essential information was recorded, including sex, age, weight, height and important history. Basal pulse oxygen saturation was observed, and unilateral intravenous access via a peripheral vein in an upper limb was established for the injection of propofol and any salvage drugs. Electrocardiographic data, pulse oxygen saturation, respiration, heart rate and blood pressure were monitored during the procedure. The nasal catheter or endoscopic mask was placed before the administration of propofol and the 
oxygen-flow rate in both groups was 5 L.min ${ }^{-1}$. Subjects were placed in the left lateral recumbent position and instructed to breathe deeply. A $1 \mathrm{mg} . \mathrm{kg}^{-1}$ propofol dose was injected intravenously within $50 \mathrm{~s}$ in the nasal catheter groups and endoscopic mask groups, with all subjects maintaining spontaneous respiration. Gastroscopy was performed after the palpebral reflex had disappeared, and an additional 30-50 mg dose of propofol was injected during the operation. If the pulse oxygen saturation decreased to $\leq 90 \%$ during the procedure, the mandible was lifted to relieve airway obstruction; if the pulse oxygen saturation decreased to $\leq 85 \%$, pressurized oxygen was supplied to guarantee the safety of the subjects. Subjects were sent to the post-anesthesia care unit (PACU) for observation for $20 \mathrm{~min}$ after painless gastroscopy and were followed up $1 \mathrm{~h}$ later.

The primary outcomes were minimum pulse oxygen saturation and incidence of pulse oxygen saturation $\leq 90 \%$. The secondary outcomes included the incidence of adverse reactions, time to examination, recovery time and propofol dosage (including basal dose and additional dose). The adverse reactions included arrhythmia, tongue retraction, cough, agitation, hiccups, reflux and aspiration. Recovery time was measured using the Observer's Assessment of Alertness/Sedation scale (OAAS, Table 1) [7, 8]. An OAAS score of 5 indicated rapid response to normal voice. The recovery time was calculated from the beginning of the procedure to the acquisition of an OAAS score of 5 in the PACU.

\section{Statistical analysis}

Continuous variables were compared using the $t$-test or Mann-Whitney $U$ test, depending on the distribution of the data. Categorical variables were compared using the Pearson chi-square test or Fisher exact test. Statistical significance was assumed at a two-sided $P$ value of $<0.05$. The results were expressed as means with SDs. The statistical analyses were performed using SPSS version 20.0 (SPSS Inc., Chicago, IL).

\section{Results}

A total of 141 patients met the inclusion criteria for painless gastroscopy, and agreed to participate in the study (Fig. 3). 7 patients were lost to follow-up. Finally, 134 aged patients were analyzed, including 69 in nasal catheter group and 65 in the endoscopic mask group. The baseline characteristics of the aged patients were balanced between the endoscopic mask and nasal catheter groups (Table 2).

Table 1. Observer's Assessment of Alertness/Sedation Scale.

\begin{tabular}{ll}
\hline 1 & Does not respond to mild prodding or shaking \\
\hline 2 & Responds to mild prodding or shaking \\
3 & Responds only after name is spoken loudly or repeatedly \\
4 & Lethargic response to name spoken in normal tone \\
5 & Responds readily to name spoken in normal tone \\
\hline
\end{tabular}

Table 2. Demographic and clinical characteristics of aged patients who underwent painless gastroscopy

\begin{tabular}{lll}
\hline & $\begin{array}{l}\text { Endoscopic mask } \\
\text { group (n=65) }\end{array}$ & $\begin{array}{l}\text { Nasal catheter } \\
\text { group (n=69) }\end{array}$ \\
\hline Age, yr (SD) & $70.6(5.6)$ & $70.1(4.4)$ \\
Male (\%) & $32(49.2)$ & $28(40.6)$ \\
Weight, kg (SD) & $58.2(11.5)$ & $59.9(10.1)$ \\
Hypertension (\%) & $13(20)$ & $17(24.6)$ \\
Diabetes (\%) & $4(6.2)$ & $6(8.7)$ \\
Arterial coronary disease (\%) & $6(9.2)$ & $6(8.7)$ \\
Cerebrovascular disease (\%) & $1(1.5)$ & $2(2.9)$ \\
Atrial septal defect (\%) & 0 & $1(1.4)$ \\
Atrial fibrillation (\%) & $3(4.6)$ & $1(1.4)$ \\
Liver cirrhosis (\%) & $1(1.5)$ & $1(1.4)$ \\
Chronic obstructive pulmonary & $1(1.5)$ & $1(1.4)$ \\
disease (\%) & & \\
Renal insufficiency (\%) & $3(4.6)$ & 0 \\
Parkinson disease (\%) & $1(1.5)$ & 0 \\
Asthma (\%) & $1(1.5)$ & 0 \\
ASA risk score (\%) & & \\
I & $33(50.8)$ & $36(52.2)$ \\
II & $32(49.2)$ & $33(47.8)$ \\
Basal SPO2, \%(SD) & $96.4(2.7)$ & $97.0(2.0)$ \\
\hline Note: SPO2, pulse oxygen saturation & ASA American Society of Anesthesiologists.
\end{tabular}

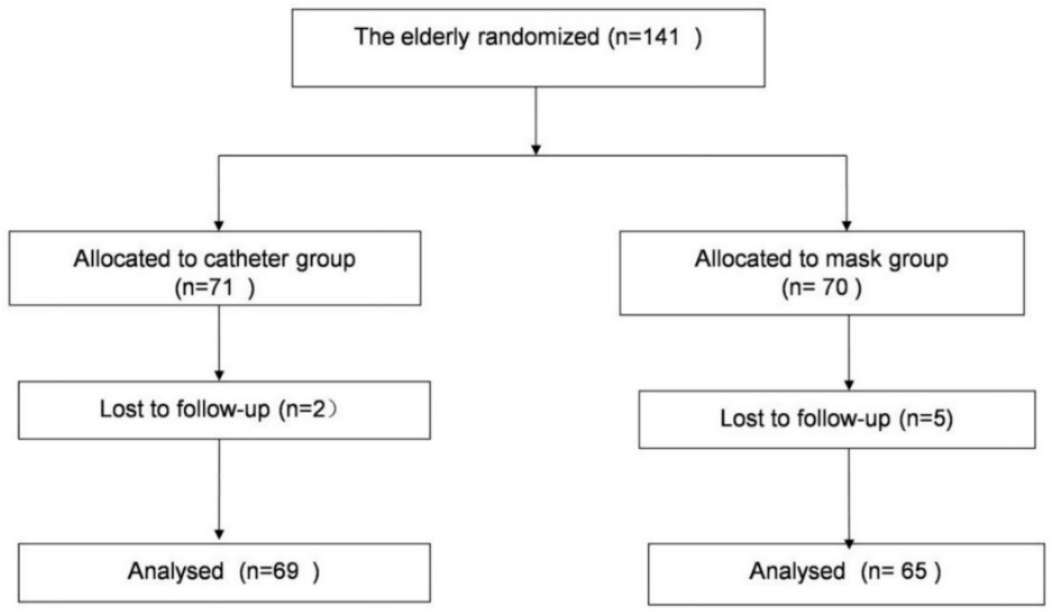

Figure 3. Flow chart of participant selection for painless gastroscopy. 
The minimum pulse oxygen saturation during painless gastroscopy was significantly higher in endoscopic mask group $(96.4 \% \pm 4.8 \%)$ than in nasal catheter group $(94.3 \% \pm 5.6 \%, P=0.0075$; Fig. 4$)$. The incidence of pulse oxygen saturation $\leq 90 \%$ did not significantly differ between the endoscopic mask group $(6.2 \%, 4 / 65)$ and nasal catheter group $(15.9 \%$, 11/69), $P=0.07$; Fig. 5)

The results demonstrated the use of endoscopic mask had no significant differences in time to examination $(P=0.70)$, recovery time $(P=0.66)$ and propofol dosage $(P=0.35)$ between the endoscopic mask group and nasal catheter group (Table 3). Similarly, incidence of cough, agitation, hiccups or arrhythmia between endoscopic mask group and nasal catheter group showed no differences (all $P>$ 0.05, Table 3).

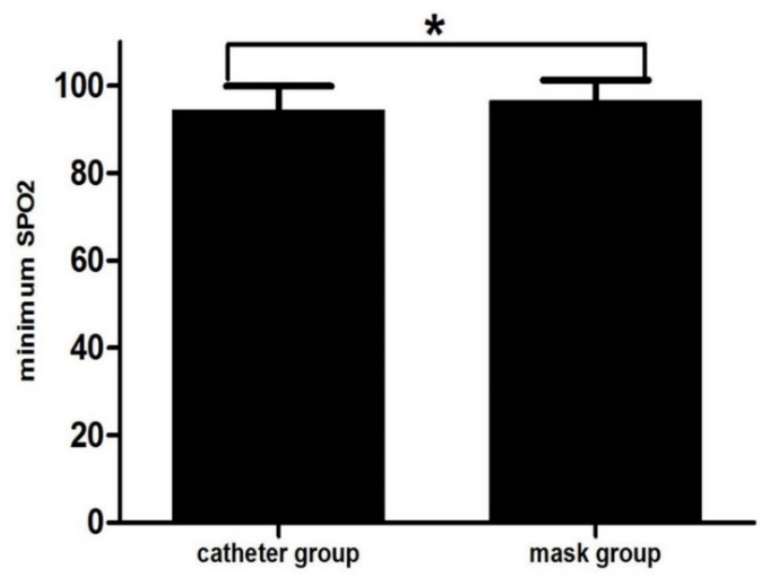

Figure 4. Minimum pulse oxygen saturation $\left(\mathrm{SPO}_{2}\right)$ during painless gastroscopy in aged patients. The minimum $\mathrm{SPO}_{2}$ was higher in the endoscopic mask group than in the nasal catheter group among aged patients $\left({ }^{*} P=0.0075\right)$.

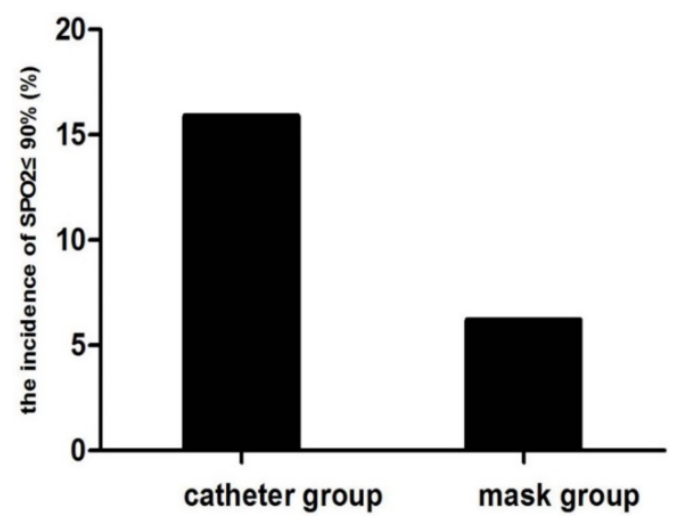

Figure 5. The incidence of SPO $2 \leq 90 \%$ during painless gastroscopy in the mask group and catheter group of aged patients.
Table 3. Secondary outcomes in aged patients who underwent painless gastroscopy

\begin{tabular}{llll}
\hline & $\begin{array}{l}\text { Endoscopic mask } \\
\text { group(n=65) }\end{array}$ & $\begin{array}{l}\text { Nasal catheter } \\
\text { group(n=69) }\end{array}$ & $P$ Value \\
\hline Time consuming, s(SD) & $280.9(131.6)$ & $272.0(132.1)$ & 0.70 \\
Recovery time, s(SD) & $581.3(258.8)$ & $600.8(248.1)$ & 0.66 \\
$\begin{array}{l}\text { Propofol dosage, mg(SD) } \\
\text { Adverse reactions }\end{array}$ & $100.3(27.6)$ & $104.9(28.6)$ & 0.35 \\
Cough (\%) & $12(18.5 \%)$ & $21(30.4 \%)$ & 0.11 \\
Agitation (\%) & $1(1.5 \%)$ & 0 & 0.49 \\
Hiccup (\%) & 0 & $1(1.4 \%)$ & 1 \\
Arrhythmia (\%) & 0 & $2(2.9 \%)$ & 0.53 \\
\hline
\end{tabular}

\section{Discussion}

The use of the endoscopic mask in aged patients during painless gastroscopy increased the minimum pulse oxygen saturation without increasing the time to examination. Several factors are considered to contribute to hypoxia during gastroscopy. The insertion of the endoscopic probe into the esophagus and stomach through the oropharynx may produce mechanical obstruction of the pharynx or compress the trachea, leading to hypoxia[3]. The use of a sedative is another important cause of oxygen desaturation. General anesthesia is likely to cause respiratory center depression and lead to hypoventilation and hypoxemia, especially when several drugs are used together[3, 9]. Gradual anesthesia induction with propofol shows a linear relationship with the augmentation of upper airway collapsibility, which can cause airway obstruction[4, 10]. Patients with cardiopulmonary diseases are more sensitive to oxygen deficiency [5]. Yazawa et al. studied 53 patients undergoing upper gastrointestinal endoscopy before cardiac surgery [6], aiming to determine the effects and risks of upper gastrointestinal procedures in patients with heart disease. Their results showed a higher incidence of oxygen desaturation in patients with a high New York Heart Association functional class. Oxygen requirements are high in aged patients because of age-related deterioration in cardiopulmonary function.

The endoscopic mask provided a new method of controlling hypoxia during painless gastroscopy. Since gastroscopy is an invasive procedure and an unpleasant experience for patients, it is increasingly being performed under sedation, and propofol is commonly used to provide sedation during gastroscopy because it is short-acting and associated with rapid recovery, leading to high patient satisfaction[11, 12]. However, oxygen desaturation and cardiac side events can occur with the use of 
propofol sedation during esophagogastroduodenoscopy (EGD)[1, 2]. For the sake of safety, a pulse oxygen saturation of $90 \%$ or less during gastroscopy is considered to indicate hypoxia and require airway intervention to improve oxygen supply and prevent respiratory depression[13, 14]. During painless gastroscopy, continuous oxygen supply and pulse oximetry are recommended for reducing the incidence of oxygen desaturation, and for the monitoring and timely detection of hypoxia, respectively[15]. However, the incidence of propofol-related hypoxia has been reported to be highly variable $(4 \%-50 \%)$ in the clinical practice guidelines of the Spanish Society of Digestive Endoscopy[15].

Compared to the routine mask, the endoscopic mask has several modifications (Fig. 1A). The endoscopic mask has a respiratory interface, an oral opening and a nasal opening in its surface. The respiratory interface is at the margin of the endoscopic mask, whereas it occupies a central position in routine masks. The oral opening is designed for gastroscope, oral tracheoscope, choledochoscope and enteroscope insertion, and the nasal opening serves as the passageway for nasal fiberoptic bronchoscopy or nasal tracheoscopy. The respiratory interface can connect to ventilation devices, and both the oral and nasal openings have valves to prevent air leakage. The endoscopic mask seals the face and can increase the fractional inspired oxygen. At the beginning of painless gastroscopy, adequate oxygen is provided through the respiratory interface. If oxygen desaturation occurs during painless gastroscopy, the respiratory interface can be connected to ventilation devices to enable safe continuation of the procedure without removing the endoscope.

A nasal cannula and mouth opener with oxygen tubing are usually used to meet oxygen requirements during gastroscopy[15]. Ventilation using conventional interfaces is a real problem during gastroscopy, since it cannot be realized without removing the endoscope[16, 17]. When severe desaturation occurs (pulse oxygen saturation $\leq 85 \%$ ), the endoscopic probe is removed to supply pressurized oxygen via a routine oxygen mask, interrupting the gastroscopy and causing inconvenience to the endoscopists and patients. The use of routine endoscopic masks can overcome this problem, as adequate oxygen supply can be delivered through the mask without removing the endoscope. However, a high-arched palate of routine endoscopic masks is not convenient for the insertion of an endoscopic probe, and routine endoscopic masks lack a nasal opening and a connector to the oropharyngeal airway. Our study showed that the use of the novel endoscopic mask in aged patients during painless gastroscopy increased the minimum pulse oxygen saturation without increasing the time to examination and recovery time. We did not conduct the study on patients older than 80 years for ethical reasons. However, the endoscopic mask has been used in several patients older than 80 years who had various complications in the clinic, with high patient and clinician satisfaction. We will design another study to estimate the effects of the endoscopic mask on patients older than 80 years with severe cardiopulmonary diseases.

The novel endoscopic mask was primarily designed to supply oxygen during painless gastroscopy, awake nasal or oral fiberoptic bronchoscopic tracheal intubation, awake nasal or oral tracheoscopy, choledochoscopy and enteroscopy. The oropharyngeal airway connecting to the endoscopic mask is a unique point and combined use with the endoscopic mask could prevent respiratory depression caused by tongue retraction, thus the endoscopic mask can be applied to patients undergoing short surgeries under general anesthesia without the use of tracheal intubation or a laryngeal mask airway or neuromuscular blocking drugs, as well as for coma patients in the NICU(Nervous System Intensive Care Unit) after being fitted with an oropharyngeal airway. However, we did not design different sizes of mask based on our novel mask fitting for demand of endoscopy in infants and young children, and we will try to improve in this area.

\section{Conclusions}

The results of this study demonstrate that the use of the endoscopic mask increased minimum pulse oxygen saturation in the aged without severe adverse events or increasing the time to examination. We recommend its routine use during painless gastroscopy in aged patients, especially, in patients with complications or difficult airways.

\section{Acknowledgments}

The promotion project of the advanced appropriate technology of Shanghai Health System (No. 2013SY032).The authors thank Shuchang $\mathrm{Xu}$, M.D, Ph.D. (Department of Gastroenterology, Tongji Hospital, Shanghai, China), Changqing Yang, M.D., Ph.D. (Department of Gastroenterology, Tongji Hospital , Shanghai, China), and Zhirong Wang, M.D, Ph.D (Department of Gastroenterology, Tongji Hospital , Shanghai, China), for their support during the study. The authors also thank all gastroscopy room nursing staff and physicians at Endoscopy Center for help and patients during the study. 


\section{Competing Interests}

The authors have declared that no competing interest exists.

\section{References}

1. Nelson DB, Barkun AN, Block KP, Burdick JS, Ginsberg GG, Greenwald DA, et al. Propofol use during gastrointestinal endoscopy. Gastrointestinal endoscopy. 2001; 53: 876-9.

2. Murray AW, Morran CG, Kenny GN, Macfarlane P, Anderson JR. Examination of cardiorespiratory changes during upper gastrointestinal endoscopy. Comparison of monitoring of arterial oxygen saturation, arterial pressure and the electrocardiogram. Anaesthesia. 1991; 46: 181-4.

3. Rimmer KP, Graham K, Whitelaw WA, Field SK. Mechanisms of hypoxemia during panendoscopy. Journal of clinical gastroenterology. 1989; 11: 17-22.

4. Hillman DR, Walsh JH, Maddison KI, Platt PR, Kirkness JP, Noffsinger WJ, et al. Evolution of changes in upper airway collapsibility during slow induction of anesthesia with propofol. Anesthesiology. 2009; 111: 63-71.

5. Seinela L, Reinikainen P, Ahvenainen J. Effect of upper gastrointestinal endoscopy on cardiopulmonary changes in very old patients. Archives of gerontology and geriatrics. 2003; 37: 25-32.

6. Yazawa K, Adachi W, Koide N, Watanabe H, Koike S, Hanazaki K. Changes in cardiopulmonary parameters during upper gastrointestinal endoscopy in patients with heart disease: towards safer endoscopy. Endoscopy. 2000; 32: 287-93

7. Kim J, Kim WO, Kim HB, Kil HK. Adequate sedation with single-dose dexmedetomidine in patients undergoing transurethral resection of the prostate with spinal anaesthesia: a dose-response study by age group. BMC Anesthesiol. 2015; 15: 17.

8. Chernik DA, Gillings $\mathrm{D}$, Laine $\mathrm{H}$, Hendler $\mathrm{J}$, Silver JM, Davidson $\mathrm{AB}$, et al. Validity and reliability of the Observer's Assessment of Alertness/Sedation Scale: study with intravenous midazolam. Journal of clinical psychopharmacology. 1990; 10: 244-51.

9. Arrowsmith JB, Gerstman BB, Fleischer DE, Benjamin SB. Results from the American Society for Gastrointestinal Endoscopy/U.S. Food and Drug Administration collaborative study on complication rates and drug use during gastrointestinal endoscopy. Gastrointestinal endoscopy. 1991; 37: 421-7.

10. Perel A. Non-anaesthesiologists should not be allowed to administer propofol for procedural sedation: a Consensus Statement of 21 European National Societies of Anaesthesia. European journal of anaesthesiology. 2011; 28: 580-4.

11. Heuss LT, Inauen W. The dawning of a new sedative: propofol in gastrointestinal endoscopy. Digestion. 2004; 69: 20-6.

12. Ferreira AO, Cravo M. Sedation in gastrointestinal endoscopy: Where are we at in 2014? World journal of gastrointestinal endoscopy 2015; 7: 102-9.

13. Allam S, Anderson KJ, O'Brien C, Macpherson JA, Gambhir S, Leitch JA, et al. Patient-maintained propofol sedation using reaction time monitoring: a volunteer safety study. Anaesthesia. 2013; 68: 154-8.

14. Charlton JE. Monitoring and supplemental oxygen during endoscopy. BMJ (Clinical research ed). 1995; 310: 886-7.

15. Igea F, Casellas JA, Gonzalez-Huix F, Gomez-Oliva C, Baudet JS, Cacho G, et al. Sedation for gastrointestinal endoscopy. Endoscopy. 2014; 46: 720-31.

16. Pisani L, Carlucci A, Nava S. Interfaces for noninvasive mechanical ventilation: technical aspects and efficiency. Minerva anestesiologica. 2012; 78: 1154-61.

17. Sferrazza Papa GF, Di Marco F, Akoumianaki E, Brochard L. Recent advances in interfaces for non-invasive ventilation: from bench studies to practical issues. Minerva anestesiologica. 2012; 78: 1146-53. 